\title{
Serum plasminogen activator urokinase receptor predicts elevated risk of acute respiratory distress syndrome in patients with sepsis and is positively associated with disease severity, inflammation and mortality
}

\author{
DAN CHEN, XIAOLING WU, JUNHUI YANG and LI YU \\ Department of Intensive Care Unit, The Central Hospital of Wuhan, Tongji Medical College, \\ Huazhong University of Science and Technology, Wuhan, Hubei 430014, P.R. China
}

Received December 12, 2018; Accepted June 13, 2019

DOI: $10.3892 /$ etm.2019.7931

\begin{abstract}
The present study aimed to evaluate the predictive value of serum soluble urokinase plasminogen activator receptor (suPAR) regarding the risk of acute respiratory distress syndrome (ARDS) in sepsis patients, and investigate its correlation/association with disease severity, inflammation and mortality in sepsis patients with ARDS. A total of 57 sepsis patients with ARDS and 58 sepsis patients without ARDS were recruited for the present case-control study. Laboratory tests, acute physiology and chronic health evaluation (APACHE) II score and sequential organ failure assessment (SOFA) score were evaluated, and mortality during hospitalization was recorded. Blood samples were collected and serum suPAR was detected by ELISA. Furthermore, tumor necrosis factor (TNF)- $\alpha$, interleukin (IL)-1 $\beta$, IL-6, IL-8, IL-10 and IL-17, as well as C-reactive protein (CRP) were detected. The results indicated that the serum levels of suPAR in sepsis patients with ARDS were higher than those in sepsis patients without ARDS. Receiver operating characteristics (ROC) curve analysis indicated that it was possible to distinguish sepsis patients with ARDS from sepsis patients without ARDS based on their serum suPAR levels, and multivariate logistic regression analysis suggested that serum suPAR levels were an independent predictor of the risk of ARDS in sepsis patients. In sepsis patients with ARDS, serum suPAR levels were positively correlated with the APACHE II score, SOFA score and the levels of CRP, TNF- $\alpha$, IL-1 $\beta$ and IL-8. In addition, serum suPAR levels were lower in survivors compared with those in non-survivors, and ROC curve analysis suggested that
\end{abstract}

Correspondence to: Dr Li Yu, Department of Intensive Care Unit, The Central Hospital of Wuhan, Tongji Medical College, Huazhong University of Science and Technology, 26 Shengli Street, Wuhan, Hubei 430014, P.R. China

E-mail: liang35575515@163.com

Key words: soluble urokinase plasminogen activator receptor, sepsis, acute respiratory distress syndrome, disease severity, mortality serum suPAR was able to predict the probability of mortality. In conclusion, serum suPAR independently predicted an elevated risk of ARDS in patients with sepsis, and was correlated/associated with greater disease severity, higher inflammation and increased mortality in patients with sepsis and ARDS.

\section{Introduction}

Sepsis is a life-threatening condition caused by unbalanced host responses to severe infection (1). Based on a global epidemiologic report, the incidence of sepsis ranges from 38 to 110 cases per 100,000 individuals, and the mortality rate is $22-55 \%$ (2). Due to systemic inflammation, it is estimated that one third of patients with sepsis develop acute respiratory distress syndrome (ARDS), a common lethal disease characterized by acute and diffuse lung injury causing severe hypoxemia (3). Despite recent improvements in critical care and the significant research effort in basic research and clinical trials on sepsis complicated with ARDS, the recovery rate remains poor and the associated mortality remains high (4). Several biomarkers, including interleukin (IL)-18, IL-15 and interferon (IFN)- $\gamma$, are known to be closely correlated with inflammation and the development of lung disease $(5,6)$. Further research into additional biomarkers is essential to predict the risk of ARDS in patients with sepsis. Such markers may be also useful for monitoring disease development in cases of sepsis-induced ARDS, which may also improve clinical outcomes.

Soluble plasminogen activator urokinase receptor (suPAR) is a $55-60 \mathrm{kDa}$ glycoprotein that occurs in three forms (I-III, II-III and I), and is the soluble form of UPAR, which is cleaved by proteases from the cell surface into its soluble form in response to inflammatory stimulation (7-9). suPAR may be detected in blood serum, urine, and bronchoalveolar lavage and cerebrospinal fluid, and its serum concentration remains stable under certain circadian changes and fasting, and due to its stability, it may be an excellent diagnostic and prognostic biomarker (10). Studies have demonstrated that suPAR may have a pro-inflammatory effect in several inflammatory diseases and, as a plasminogen activator, it has been demonstrated to facilitate coagulation as well as fibrinolytic cascades $(11,12)$. 
Overexpression of suPAR has also been reported to be associated with a high risk of 30-day mortality in sepsis patients (13). Furthermore, its upregulation was demonstrated to be positively correlated with higher disease severity in patients with ARDS (14). Considering the association of suPAR with the severity of ARDS and its potential value as a prognostic indicator, the present study hypothesized that suPAR may have a critical role in patients with sepsis complicated with ARDS. Therefore, the present study aimed to evaluate the predictive value of serum suPAR regarding the risk of ARDS in patients with sepsis, and to investigate the correlation of suPAR with disease severity, inflammation and mortality.

\section{Materials and methods}

Patients. Between January 2015 and December 2017, 57 patients with sepsis complicated by ARDS treated at The Central Hospital of Wuhan, Tongji Medical College, Huazhong University of Science and Technology (Wuhan, China) were recruited for the present study. The inclusion criteria were as follows: i) Diagnosis with sepsis according to the 2001 International Sepsis Definitions Conference criteria (15); ii) complication of ARDS, which was diagnosed in accordance with the Berlin criteria (16); iii) age $>18$ years. Patients were excluded if the following applied: i) No inflammatory status; ii) other complications/malignancies; iii) human immunodeficiency virus infection; iv) pregnancy. None of the sepsis patients received any treatments for sepsis prior to enrolment in the present study. During the same period, patients diagnosed with sepsis without the complication of ARDS, who were admitted to the hospital's intensive care unit (ICU) and did not meet any of the exclusion criteria described for patients with ARDS, were recruited for the present study. Thereby, a total of 58 patients with sepsis and without ARDS, who were ageand gender-matched with the ARDS group, were selected as a control group. The present study was approved by the Ethics Committee of The Central Hospital of Wuhan, Tongji Medical College, Huazhong University of Science and Technology (Wuhan, China), and all participants or their guardians provided written informed consent prior to enrollment.

Data collection. Clinical data for each patient, including age, gender, body mass index (BMI), history of smoking and chronic comorbidities [including chronic obstructive pulmonary disease (COPD), cardiomyopathy, chronic kidney failure and cirrhosis] of all patients were recorded in a case report form after enrollment. For the patients with ARDS, laboratory tests [including serum creatinine (Scr), albumin, white blood cells (WBC), C-reactive protein (CRP) and procalcitonin (PCT)] were performed within $24 \mathrm{~h}$ after the onset of ARDS, and the acute physiology and chronic health evaluation (APACHE) II score and sequential organ failure assessment (SOFA) score were evaluated at the same time. As for the patients without ARDS, the laboratory tests were performed within $24 \mathrm{~h}$ after ICU admission, and their APACHE II score and SOFA score were also assessed. The APACHE II score value was calculated based on the initial scoring of 12 components with a range of 0 to 71 points, and a higher score was associated with higher severity (17). In the SOFA scoring scheme, 1-4 points were assigned regarding the dysfunction levels of six organ systems (respiratory, circulatory, renal, hematological, hepatic and central nervous systems), resulting in a total score of 0-24 points, with higher scores being associated with higher severity (18). The patients were followed up until discharge from hospital and mortality during hospitalization was recorded.

Blood sample collection. Blood samples of the patients with ARDS were collected within $24 \mathrm{~h}$ after the occurrence of ARDS. As for the patients without ARDS, blood samples were extracted within $24 \mathrm{~h}$ after ICU admission. All samples were collected in serum separator tubes, allowed to clot for $30 \mathrm{~min}$ and then immediately centrifuged for $15 \mathrm{~min}$ at $2,000 \mathrm{x} \mathrm{g}$ at $4^{\circ} \mathrm{C}$ to separate the serum. Subsequently, the serum was aliquoted and stored at $-20^{\circ} \mathrm{C}$ until use.

Measurement of SUPAR and inflammatory cytokines. The serum suPAR levels of all patients were detected using a commercial ELISA kit (cat. no. ml060583; Shanghai Enzyme-linked Biotechnology Co., Ltd.), according to the manufacturer's protocol. The levels of inflammatory cytokines, including tumor necrosis factor (TNF)- $\alpha$, IL-1 $\beta$, IL-6 (cat. no. ml058097), IL-8 (cat. no. ml028580), IL-10 (cat. no. ml064299), IL-17 (cat. no. ml058051), IL-1 $\beta$ (cat. no. m1058059) and TNF- $\alpha$ (cat. no. m1077385) in the blood serum were also measured using human ELISA kits (Shanghai Enzyme-linked Biotechnology Co., Ltd.), according to the manufacturer's protocols.

Statistical analysis. SPSS 22.0 (IBM Corp.) and GraphPad Prism 7.0 (GraphPad Software, Inc.) were used for statistical data processing and generation of figures. Shapiro-Wilk test was used to determine the normality of continuous variables. Normally distributed continuous variables were expressed as the mean \pm standard deviation, non-normally distributed continuous data were presented as the median (25th-75th quartiles) and categorical variables were expressed as n (\%). Comparisons of normally distributed continuous variables between groups were performed using Student's t-test, differences in non-normally distributed continuous variables between groups were determined by Wilcoxon's signed-rank sum test and comparisons of categorical variables between groups were performed by Chi-squared tests. Correlation analyses were performed using Spearman's test. Receiver-operating characteristic (ROC) curves were drawn and the area under the ROC curve (AUC) was determined to assess the feasibility of serum suPAR as a potential biomarker to predict the risk of ARDS in patients with sepsis, as well as to assess its value in predicting the risk of mortality. Univariate and multivariate logistic regression model analyses were used to determine the value of suPAR in predicting the risk of ARDS in patients with sepsis. Sepsis severity was assessed by the correlation analysis of suPAR, which was performed using the Spearman test. $\mathrm{P}<0.05$ was considered to indicate statistical significance.

\section{Results}

Baseline characteristics. The mean age of the patients with sepsis without ARDS $(n=58)$ and with ARDS $(n=57)$ was 
Table I. Baseline characteristics of patients with sepsis with/without ARDS.

\begin{tabular}{|c|c|c|c|}
\hline Characteristics & Sepsis without ARDS $(\mathrm{n}=58)$ & Sepsis with ARDS $(n=57)$ & P-value \\
\hline Age (years) & $57.6 \pm 9.2$ & $56.3 \pm 10.1$ & 0.488 \\
\hline Gender (male/female) & $40 / 18$ & $40 / 17$ & 0.888 \\
\hline $\operatorname{BMI}\left(\mathrm{kg} / \mathrm{m}^{2}\right)$ & $23.0 \pm 4.4$ & $23.0 \pm 4.7$ & 0.978 \\
\hline History of smoking & $14(24.1)$ & $29(50.9)$ & 0.003 \\
\hline \multicolumn{4}{|l|}{ Chronic comorbidities } \\
\hline COPD & $8(13.8)$ & $12(21.1)$ & 0.304 \\
\hline Cardiomyopathy & $22(37.9)$ & $22(38.6)$ & 0.941 \\
\hline Cardiovascular disease & $23(39.7)$ & $24(42.1)$ & 0.789 \\
\hline Type 2 diabetes & $9(15.5)$ & $11(19.3)$ & 0.593 \\
\hline Chronic kidney failure & $6(10.3)$ & $4(7.0)$ & 0.763 \\
\hline Cirrhosis & $10(17.2)$ & $9(15.8)$ & 0.834 \\
\hline $\operatorname{Scr}(\mathrm{mg} / \mathrm{dl})$ & $1.4(0.9-2.1)$ & $1.4(1.1-2.0)$ & 0.535 \\
\hline Albumin (g/l) & $26.9(22.4-36.8)$ & $28.7(21.3-39.0)$ & 0.873 \\
\hline WBC $\left(10^{9} / 1\right)$ & $12.5(3.1-28.6)$ & $12.3(3.9-26.3)$ & 0.851 \\
\hline CRP (mg/l) & $83.1(53.4-132.4)$ & $79.7(53.3-162.8)$ & 0.535 \\
\hline PCT (ng/ml) & $14.1(8.5-21.6)$ & $19.9(8.7-29.1)$ & 0.170 \\
\hline APACHE II score & $15.2 \pm 5.6$ & $17.4 \pm 4.5$ & 0.018 \\
\hline SOFA score & $8.3 \pm 4.0$ & $9.6 \pm 3.9$ & 0.082 \\
\hline TNF- $\alpha(p g / m l)$ & 148.7 (80.4-225.5) & $159.0(98.9-226.1)$ & 0.437 \\
\hline IL-1 $\beta(p g / m l)$ & $9.6(4.4-18.9)$ & $10.3(4.2-23.0)$ & 0.821 \\
\hline IL-6 (pg/ml) & $115.2(77.3-226.6)$ & $164.5(79.6-225.3)$ & 0.595 \\
\hline IL-8 (pg/ml) & $199.4(113.0-380.2)$ & $212.9(132.6-337.0)$ & 0.623 \\
\hline IL-10 (pg/ml) & $14.2(9.1-22.9)$ & $15.4(8.3-26.4)$ & 0.871 \\
\hline IL-17 (pg/ml) & $136.6(67.4-214.8)$ & $154.2(105.3-228.0)$ & 0.238 \\
\hline
\end{tabular}

Values are expressed as the mean value \pm standard deviation, $\mathrm{n}(\%)$ or median (25th-75th quartiles). Comparisons of normally distributed continuous variables between groups were performed using Student's t-test, comparisons of non-normally distributed continuous data between groups were determined by Wilcoxon's signed-rank sum test and comparisons of categorical variables between groups (including gender, smoking and chronic comorbidities) were determined with the Chi-squared test. ARDS, acute respiratory distress syndrome; BMI, body mass index; COPD, chronic obstructive pulmonary disease; Scr, serum creatinine; WBC, white blood cells; CRP, C-reactive protein; PCT, procalcitonin; APACHE, acute physiology and chronic health evaluation; SOFA, sequential organ failure assessment; TNF, tumor necrosis factor; IL, interleukin.

$57.6 \pm 9.2$ and $56.3 \pm 10.1$ years [Patients with sepsis without ARDS: Age range, 39.0-79.0 years (median age, 56.5 years); Patients with sepsis with ARDS: Age range, 31.0-82.0 years (median age, 56.0 years)], respectively (Table I). The number of patients with ARDS with a history of smoking was higher compared with that in the control group $(\mathrm{P}=0.003)$, and the APACHE II score was increased in patients with ARDS compared with that in patients without ARDS $(\mathrm{P}=0.018)$. In addition, the SOFA score was numerically higher in patients with ARDS compared with that in patients without ARDS, but this difference was not significant $(\mathrm{P}=0.082)$. No differences in any other demographic parameters, clinical features or laboratory test indexes were identified between the two groups (Table I).

Serum suPAR levels predict an elevated risk of ARDS in patients with sepsis. The median serum suPAR levels in patients with ARDS were higher compared with those in the control group [15.17 (12.77-18.01) vs. 13.14 (10.78-15.8) ng/ml; $\mathrm{P}=0.005$; Fig. 1A]. The ROC curve revealed that the serum suPAR levels were able to distinguish patients with sepsis and
ARDS from sepsis patients without ARDS (AUC, 0.651; 95\% CI, 0.550-0.751; Fig. 1B). The sensitivity and specificity were 63.2 and $63.8 \%$, respectively. In addition, the serum suPAR level was $14.01 \mathrm{ng} / \mathrm{ml}$ at the best cut-off point, where the sum of sensitivity and specificity reached its maximum value.

Factors affecting the occurrence of ARDS in patients with sepsis. In order to explore the factors affecting the occurrence of ARDS in patients with sepsis, a logistic regression analysis was performed (Table II). Univariate logistic regression analysis revealed that smoking $(\mathrm{P}=0.004)$, high serum suPAR expression $(\mathrm{P}=0.007)$ and high APACHE II scores $(\mathrm{P}=0.020)$ were associated with a higher incidence of ARDS in patients with sepsis. Further multivariate logistic regression analysis demonstrated that smoking $(\mathrm{P}=0.020)$ and high serum suPAR expression $(\mathrm{P}=0.045)$ are independent predictors of elevated risk of ARDS in patients with sepsis.

Correlation of serum suPAR levels with APACHE II score, SOFA score and levels of inflammatory factors in patients 

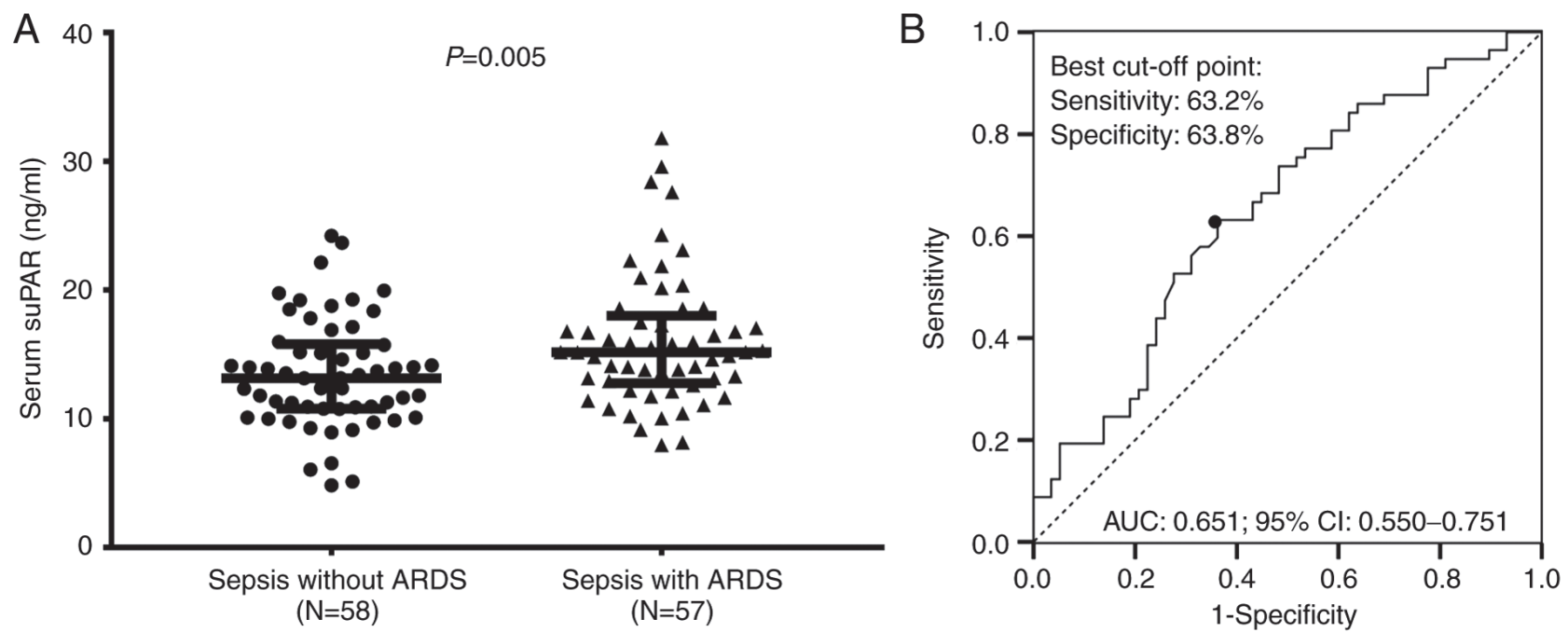

Figure 1. (A) Sepsis patients with ARDS had higher serum suPAR levels compared to sepsis patients with ARDS. (B) Serum suPAR levels were able to predict the risk of ARDS in sepsis patients, with the best cut-off set at $14.01 \mathrm{ng} / \mathrm{ml}$. SuPAR, soluble urokinase plasminogen activator receptor; ARDS, acute respiratory distress syndrome; AUC, area under curve.
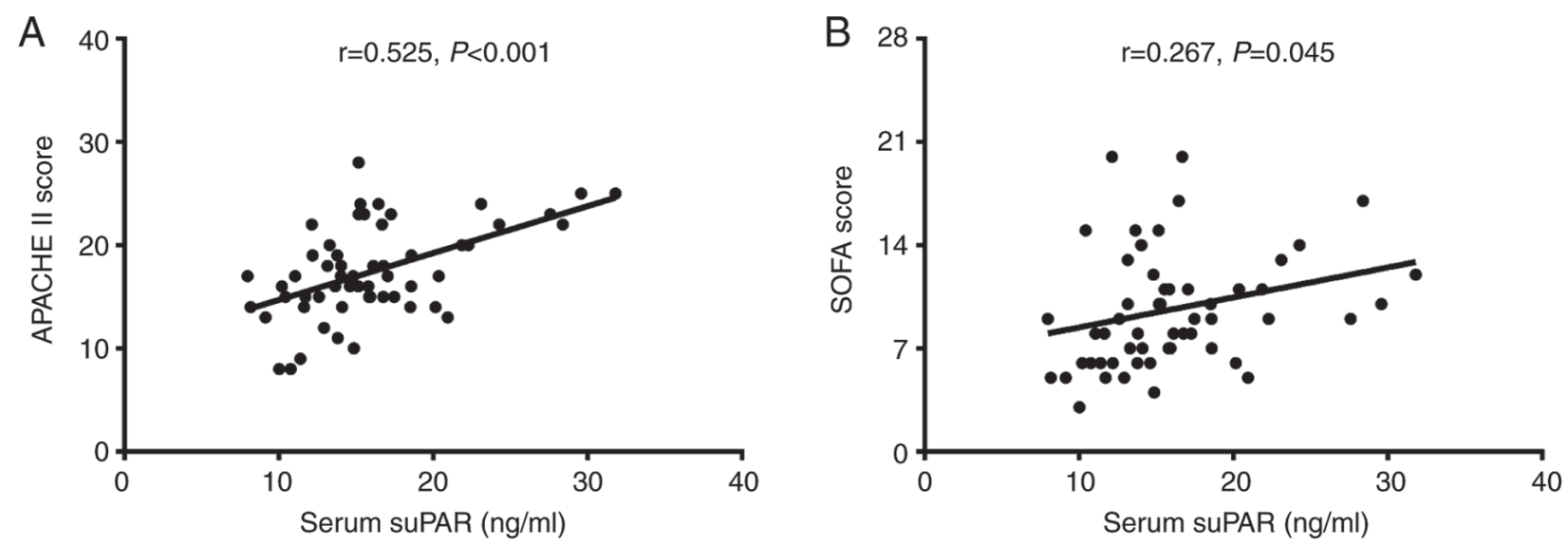

Figure 2. Correlation of serum suPAR levels with APACHE II score and SOFA score in sepsis patients with ARDS. (A) Serum suPAR levels were positively correlated with the APACHE II score in sepsis-ARDS patients. (B) Serum suPAR levels were positively correlated with the SOFA score in sepsis-ARDS patients. Correlation analysis was performed using the Spearman test. SuPAR, soluble urokinase plasminogen activator receptor; APACHE, acute physiology and chronic health evaluation; SOFA, sequential organ failure assessment.

with ARDS. In patients with ARDS, the serum suPAR levels were positively correlated with the APACHE II score $(r=0.525$, $\mathrm{P}<0.001$; Fig. 2A) and the SOFA score $(\mathrm{r}=0.267, \mathrm{P}=0.045$; Fig. 2B). Regarding their association with the levels of inflammatory factors (Fig. 3), serum suPAR levels were positively correlated with the levels of CRP ( $\mathrm{r}=0.454$, $\mathrm{P}<0.001$; Fig. 3A), TNF- $\alpha$ ( $r=0.418, P=0.001$; Fig. 3C), IL-1 $\beta$ ( $r=0.334, P=0.011$; Fig. 3D) and IL-8 (r=0.315, $\mathrm{P}=0.017$; Fig. 3F), and negatively correlated with IL-10 levels ( $r=-0.309, P=0.019$; Fig. $3 G)$ in patients with sepsis and ARDS. However, there was no correlation of serum suPAR levels with PCT levels $(\mathrm{r}=0.242, \mathrm{P}=0.070$; Fig. 3B), IL-6 levels ( $r=0.161, P=0.232$; Fig. 3E) or IL-17 levels $(\mathrm{r}=0.156, \mathrm{P}=0.246$; Fig. $3 \mathrm{H})$ in patients with sepsis and ARDS.

Serum SUPAR levels are a good predictor of survival in patients with sepsis complicated with ARDS. The cohort of patients with sepsis and ARDS comprised 23 non-survivors and 34 survivors. The median serum suPAR levels in non-survivors and survivors were 17.5 (13.8-22.3) and 14.7 (12.0-16.5) ng/ml, respectively ( $\mathrm{P}=0.023$; Fig. 4A). ROC curve analysis indicated that serum suPAR levels were capable of distinguishing survivors from non-survivors, with an AUC of 0.679 (95\% confidence interval, $0.529-0.829)$ in patients with ARDS (Fig. 4B). The sensitivity and specificity were 91.2 and $52.2 \%$, respectively. Furthermore, the serum suPAR level of $17.38 \mathrm{ng} / \mathrm{ml}$ was the best cut-off point.

\section{Discussion}

The results of the present study may be summarized as follows: i) Serum suPAR levels were higher in patients with sepsis with ARDS compared with those in patients with sepsis without ARDS, and were an independent predictor of an elevated risk of ARDS; ii) serum suPAR levels were positively correlated with the APACHE II score, the SOFA score and levels of certain pro-inflammatory factors in patients with ARDS; iii) serum 
Table II. Factors associated with a risk of ARDS in sepsis determined by logistic regression analysis.

\begin{tabular}{|c|c|c|c|c|c|c|c|c|}
\hline \multirow[b]{3}{*}{ Factors } & \multicolumn{4}{|c|}{ Univariate logistic regression } & \multicolumn{4}{|c|}{ Multivariate logistic regression } \\
\hline & \multirow[b]{2}{*}{ P-value } & \multirow[b]{2}{*}{ OR } & \multicolumn{2}{|c|}{$95 \% \mathrm{CI}$} & \multirow[b]{2}{*}{ P-value } & \multirow[b]{2}{*}{ OR } & \multicolumn{2}{|c|}{$95 \% \mathrm{CI}$} \\
\hline & & & Lowest & Highest & & & Lowest & Highest \\
\hline Age & 0.484 & 0.986 & 0.949 & 1.025 & 0.396 & 0.979 & 0.932 & 1.028 \\
\hline Gender (male vs. female) & 0.888 & 1.059 & 0.478 & 2.344 & 0.524 & 1.376 & 0.515 & 3.676 \\
\hline BMI & 0.978 & 1.001 & 0.923 & 1.086 & 0.888 & 1.008 & 0.904 & 1.124 \\
\hline Smoke (yes vs. no) & 0.004 & 3.255 & 1.471 & 7.205 & 0.020 & 3.017 & 1.189 & 7.654 \\
\hline \multicolumn{9}{|l|}{ Chronic comorbidities } \\
\hline COPD (yes vs. no) & 0.307 & 1.667 & 0.625 & 4.446 & 0.366 & 1.733 & 0.526 & 5.717 \\
\hline Cardiomyopathy (yes vs. no) & 0.941 & 1.029 & 0.485 & 2.182 & 0.452 & 1.463 & 0.543 & 3.942 \\
\hline Chronic kidney failure (yes vs. no) & 0.529 & 0.654 & 0.174 & 2.453 & 0.614 & 0.633 & 0.107 & 3.749 \\
\hline Cirrhosis (yes vs. no) & 0.834 & 0.900 & 0.336 & 2.411 & 0.450 & 0.621 & 0.180 & 2.140 \\
\hline Serum suPAR & 0.007 & 1.129 & 1.034 & 1.233 & 0.045 & 1.131 & 1.002 & 1.277 \\
\hline Scr & 0.756 & 0.955 & 0.714 & 1.277 & 0.306 & 0.791 & 0.506 & 1.238 \\
\hline Albumin & 0.874 & 0.997 & 0.959 & 1.036 & 0.662 & 0.986 & 0.924 & 1.052 \\
\hline WBC & 0.974 & 1.000 & 0.974 & 1.025 & 0.836 & 1.004 & 0.963 & 1.048 \\
\hline CRP & 0.315 & 1.003 & 0.997 & 1.008 & 0.432 & 0.994 & 0.978 & 1.010 \\
\hline PCT & 0.255 & 1.015 & 0.989 & 1.042 & 0.475 & 1.020 & 0.966 & 1.077 \\
\hline APACHE II score & 0.020 & 1.094 & 1.014 & 1.179 & 0.203 & 1.090 & 0.955 & 1.244 \\
\hline SOFA score & 0.085 & 1.087 & 0.989 & 1.195 & 0.940 & 1.006 & 0.864 & 1.170 \\
\hline TNF- $\alpha$ & 0.448 & 1.001 & 0.998 & 1.004 & 0.486 & 1.002 & 0.996 & 1.009 \\
\hline IL-1 $\beta$ & 0.641 & 1.008 & 0.976 & 1.041 & 0.962 & 1.001 & 0.945 & 1.061 \\
\hline IL-6 & 0.886 & 1.000 & 0.997 & 1.003 & 0.390 & 0.998 & 0.993 & 1.003 \\
\hline IL-8 & 0.636 & 1.000 & 0.999 & 1.002 & 0.902 & 1.000 & 0.996 & 1.003 \\
\hline IL-10 & 0.982 & 1.000 & 0.989 & 1.012 & 0.698 & 1.003 & 0.988 & 1.018 \\
\hline IL-17 & 0.131 & 1.002 & 0.999 & 1.005 & 0.082 & 1.003 & 1.000 & 1.007 \\
\hline
\end{tabular}

Factors affecting ARDS in sepsis were determined by univariate and multivariate logistic regression analyses. ARDS, acute respiratory distress syndrome; OR, odds ratio; CI, confidence interval; BMI, body mass index; COPD, chronic obstructive pulmonary disease; suPAR, soluble urokinase plasminogen activator receptor; Scr, serum creatinine; WBC, white blood cell; CRP, C-reactive protein; PCT, procalcitonin; APACHE, acute physiology and chronic health evaluation; SOFA, sequential organ failure assessment; TNF, tumor necrosis factor; IL, interleukin.

suPAR levels (collected within $24 \mathrm{~h}$ after the occurrence of ARDS) were able to distinguish survivors from non-survivors (in-hospital mortality) in the sepsis+ARDS group of patients.

suPAR, a protein derived from the cleavage of the cell membrane-bound UPAR, is involved in various biological and pathological processes, including inflammatory response, immune activation, signal transduction and tissue remodeling $(7,10,19-24)$. Due to its role in inflammation, suPAR has been reported to not only possess direct chemotactic properties to promote recruitment of inflammatory cells (including monocytes and neutrophils), but has also been reported to suppress neutrophil efferocytosis and obstruct the engulfment of apoptotic neutrophils, leading to the dysregulation of the host's immune response (25-28). In addition, suPAR has been reported to act as a plasminogen activator, facilitating coagulation and fibrinolytic cascades (11).

Recently, suPAR has been reported to be upregulated in several inflammatory diseases, including inflammatory bowel disease, arthritis and central nervous system infections (29-31). For instance, suPAR is highly expressed in patients with
Crimean-Congo hemorrhagic fever (CCHF) compared to healthy controls, and it is able to distinguish CCHF patients from healthy controls with an AUC of 0.94 (32). Another study including 273 critically ill patients with sepsis revealed that the concentration of serum suPAR is higher in these patients compared with that in healthy controls, and the AUC for sepsis prediction was 0.62 (12). Of note, suPAR may also be an emerging biomarker for patients with lung diseases. Previous studies have revealed that it is also highly expressed in patients with COPD, asthma and inhalation trauma (33-35). Considering that suPAR may be involved in the development of inflammatory diseases and lung injury-associated pathologies, it was hypothesized that suPAR may have an important role in the development of ARDS in patients with sepsis.

The present study demonstrated that suPAR is highly expressed in the serum of patients with sepsis with ARDS complications compared with patients diagnosed with sepsis but without ARDS, and it is an independent predictor of an elevated risk of ARDS in sepsis patients. ARDS may develop due to suPAR-mediated inhibition of neutrophil efferocytosis 

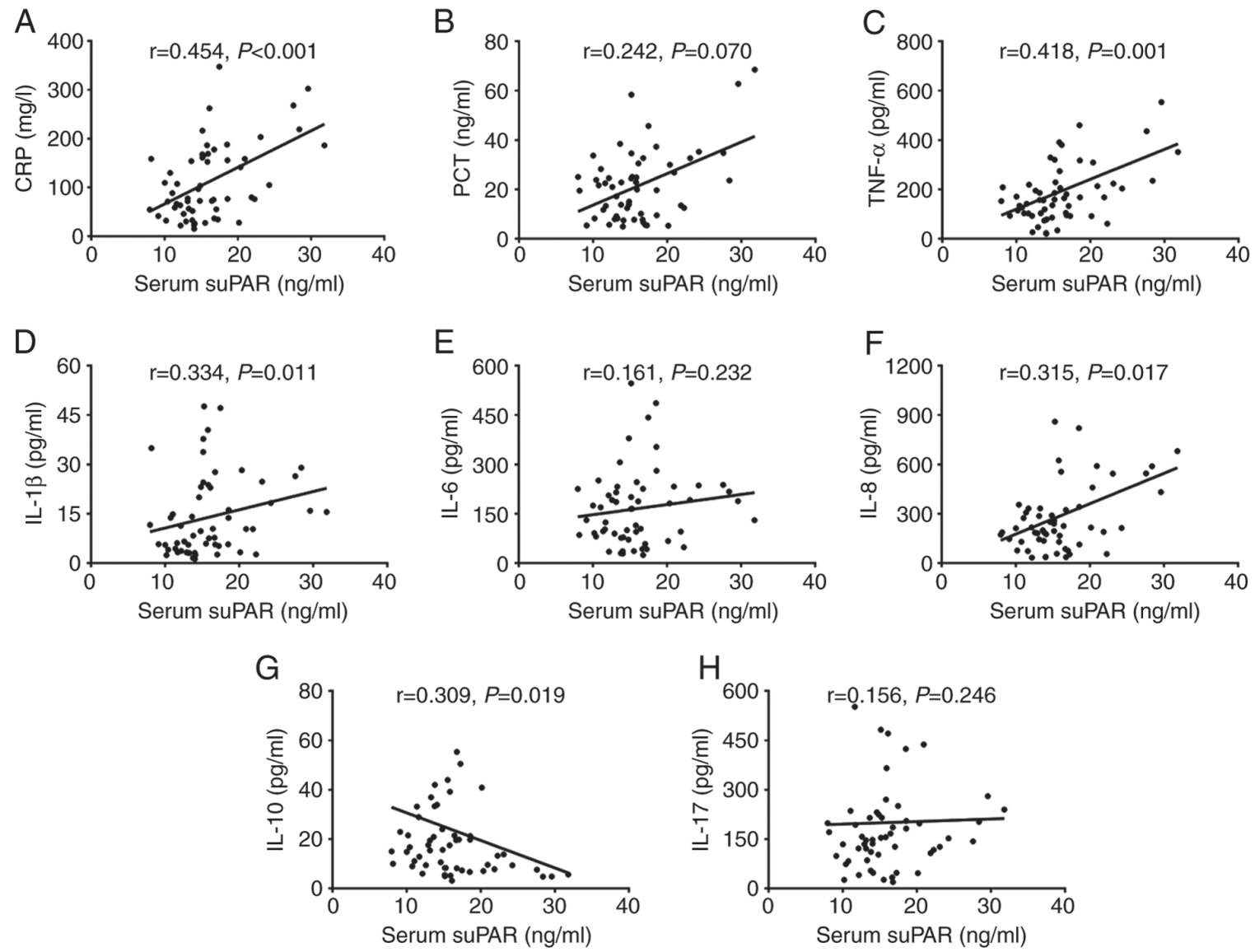

Figure 3. Correlation of the serum levels of suPAR with those of inflammatory factors (A) CRP, (B) PCT, (C) TNF- $\alpha$, (D) IL-1 3 , (E) IL-6, (F) IL-8, (G) IL-10, (H) IL-17 in sepsis-ARDS patients. Correlation analysis was performed using the Spearman test. CRP, C-reactive protein; PCT, procalcitonin; TNF, tumor necrosis factor; IL, interleukin; suPAR, soluble urokinase plasminogen activator receptor.
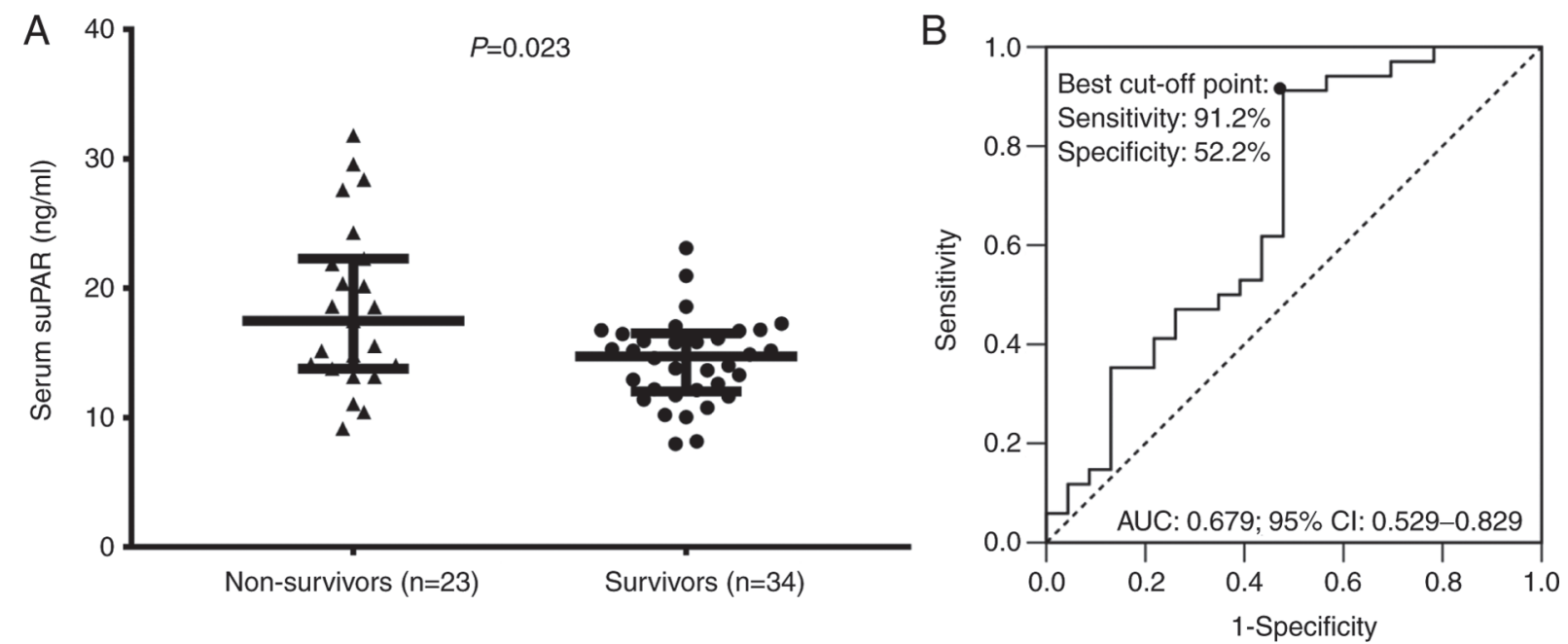

Figure 4. (A) Comparison of serum suPAR levels between non-survivors and survivors among patients with sepsis and ARDS. Non-survivors had higher serum suPAR levels than survivors. (B) Serum suPAR levels were a good prognostic predictor for survival in sepsis-ARDS patients, and the best cut-off was set at $17.38 \mathrm{ng} / \mathrm{ml}$. SuPAR, soluble urokinase plasminogen activator receptor; ARDS, acute respiratory distress syndrome; AUC, area under curve.

and obstruction of apoptotic neutrophil engulfment, which may weaken the host defenses, thus leading to a higher risk of ARDS in patients with sepsis $(25,26)$. In addition, suPAR may stimulate the recruitment of inflammatory cells (including monocytes and neutrophils), promoting inflammation, and accelerate coagulation via fibrinolytic cascades, achieved by activation of the plasminogen pathway. In turn, this process may induce acute lung injury and increase the risk of ARDS in patients with sepsis $(27,28)$. However, the AUC value, as well as the sensitivity and specificity, were relatively low in the ROC analysis, and the predictive value of the clinical parameters was also low, and only three factors (age, smoking history and 
COPD) were identified as good predictive factors for the risk of ARDS. Despite these low AUCs, as well as sensitivity and specificity values, the predictive value of suPAR for the risk of ARDS remained high. Therefore, studies with more patients and further subgroup analyses are required to explore which subgroup has relatively higher sensitivity and specificity values.

Several studies have investigated the correlation of suPAR with disease severity and inflammation in patients with inflammatory or lung diseases. In critically ill patients with sepsis, serum suPAR levels were observed to be positively correlated with acute physiology score (SAPS) II, APACHE II and SOFA scores (12). Another recent study also revealed that the levels of suPAR are associated with the SAPS II score, the APACHE II score and the concentration of pro-inflammatory factors (including TNF- $\alpha$ and CRP) in patients with sepsis (12). A study including 632 patients with ARDS revealed that higher suPAR levels are associated with more severe ARDS (14). The present study also indicated that serum suPAR levels were positively correlated with the APACHE II score, SOFA score and the levels of pro-inflammatory factors, including CRP, TNF- $\alpha$, IL- $1 \beta$ and IL-8, in patients with sepsis and ARDS, which may be explained by the facilitation of the inflammatory response by suPAR. Therefore, due to suPAR-mediated inhibition of engulfment of apoptotic neutrophils and bacteria, multiple organ dysfunction (including lung, liver and kidney) may occur following an increase in suPAR levels, leading to severe inflammation and increased disease severity in patients with sepsis and ARDS. In addition, suPAR may regulate several genes or proteins (including $\beta 3$ integrin and glycosyl-phosphatidylinositol protein), thus affecting the levels of inflammatory factors and contributing to increased inflammation and higher disease severity (36).

In clinical practice, biomarkers are used not only for diagnosing a pathological condition and distinguishing disease severity, but also for their ability to predict morbidity and clinical outcomes (37). In addition, overexpression of suPAR is associated with poorer prognosis in patients with systemic inflammation or infection $(13,14,33,36,38)$. For instance, in sepsis patients, serum suPAR independently predicted a higher risk of 30-day mortality (13). As for ARDS patients, serum suPAR levels were increased in non-survivors compared to survivors, with serum suPAR exhibiting an AUC for predicting ARDS-associated mortality of 0.62 (14). These previous studies indicated that suPAR may have a potential role in predicting a poorer prognosis in patients with sepsis, with or without ARDS complications. In the present study, serum suPAR levels were also higher in non-survivors compared with those in survivors, and they were able to distinguish survivor from non-survivor patient groups. This further strengthened the possibility that suPAR is associated with higher disease severity, resulting in a poorer prognosis of patients with sepsis complicated with ARDS. Furthermore, higher suPAR levels may also be associated with higher treatment resistance, thereby contributing to the poorer prognosis. However, this possibility requires further study and validation. A previous study evaluated the last two uric acid measurements (taken prior to discharge or mortality) in patients with sepsis or ARDS, and revealed that uric acid, one of the major non-enzymatic anti-oxidants in the blood, serves as a useful biomarker predicting worse clinical outcomes of patients with sepsis or ARDS with an AUC of 0.714 (95\%CI: 0.615-0.813) (37). In comparison, the prognostic value of suPAR in the present study was relatively lower with an AUC of 0.679 (95\% CI: 0.529-0.829) in sepsis patients with ARDS, which may be due to different patients and the relatively small sample size of the present study. However, suPAR still had a certain prognostic value in sepsis patients with ARDS, and the results of the present study may provide a novel perspective and evidence regarding the role of suPAR in sepsis patients with ARDS.

The present study had certain limitations. First, the number of patients with sepsis complicated with ARDS was matched with that of patients diagnosed with sepsis but exhibiting no symptoms of ARDS. Thus, the incidence of ARDS was not properly evaluated. Furthermore, the sample size was relatively small, which may have decreased the statistical power of the results of the present study. Hence, additional studies with large sample sizes are required for further validation of the present results. In addition, all patients were from one single hospital, and studies including more patients from multiple centers may be beneficial to deepen the understanding of this pathology. The mechanistic roles of suPAR in sepsis complicated with ARDS remain to be fully elucidated, and further experiments are required. Finally, considering that suPAR is a soluble form of uPAR, the study of UPAR levels may also provide useful insight into the development of this pathology, and may also be a good biomarker for the prediction of clinical outcomes of patients with sepsis or ARDS.

In conclusion, the present study demonstrated that serum suPAR was an independent predictor of an elevated risk of ARDS in patients with sepsis, and it was positively correlated/associated with higher disease severity, higher inflammation and increased mortality in patients with sepsis complicated with ARDS.

\section{Acknowledgements}

Not applicable.

\section{Funding}

No funding was received.

\section{Availability of data and materials}

The datasets used and/or analyzed during the current study are available from the corresponding author on reasonable request.

\section{Authors' contributions}

LY designed the study, DC, XW and JY performed the experiments, and XW and LY analyzed the data. DC, XW, JY and LY wrote the manuscript and revised it.

\section{Ethics approval and consent to participate}

The study was performed according to the established tenets in the Declaration of Helsinki and approved by the Ethics Committee of The Central Hospital of Wuhan, Tongji Medical College, Huazhong University of Science and Technology (Wuhan, China). Written informed consent was obtained from all patients or their guardians. 


\section{Patient consent for publication}

Not applicable.

\section{Competing interest}

The authors declare that they have no competing interests.

\section{References}

1. Deutschman CS and Tracey KJ: Sepsis: Current dogma and new perspectives. Immunity 40: 463-475, 2014.

2. Kempker JA and Martin GS: The changing epidemiology and definitions of sepsis. Clin Chest Med 37: 165-179, 2016.

3. Fein AM and Calalang-Colucci MG: Acute lung injury and acute respiratory distress syndrome in sepsis and septic shock. Crit Care Clin 16: 289-317, 2000.

4. Sheu CC, Gong MN, Zhai R, Chen F, Bajwa EK, Clardy PF, Gallagher DC, Thompson BT and Christiani DC: Clinical characteristics and outcomes of sepsis-related vs non-sepsis-related ARDS. Chest 138: 559-567, 2010.

5. Wu SQ, Liang W, Wang GL, Li LY, Wang DL and Chen C: Polymorphisms of the IL-18 promoter and bronchial asthma. Mol Med Rep 6: 1385-1388, 2012.

6. Grzesk E, Koltan S,Debski R, Wysocki M, Gruszka M,KubickaM, Koltan A, Grzesk G, Manysiak S and Odrowaz-Sypniewska G: Concentrations of IL-15, IL-18, IFN- $\gamma$ and activity of CD4 $4^{+}$ $\mathrm{CD}^{+}$and $\mathrm{NK}$ cells at admission in children with viral bronchiolitis. Exp Ther Med 1: 873-877, 2010.

7. Thuno M, Macho B and Eugen-Olsen J: suPAR: The molecular crystal ball. Dis Markers 27: 157-172, 2009.

8. Mekonnen G, Corban MT, Hung OY, Eshtehardi P, Eapen DJ, Al-Kassem H, Rasoul-Arzrumly E, Gogas BD, McDaniel MC, Pielak T, et al: Plasma soluble urokinase-type plasminogen activator receptor level is independently associated with coronary microvascular function in patients with non-obstructive coronary artery disease. Atherosclerosis 239: 55-60, 2015

9. Tzanakaki G, Paparoupa M, Kyprianou M, Barbouni A, Eugen-Olsen J and Kourea-Kremastinou J: Elevated soluble urokinase receptor values in CSF, age and bacterial meningitis infection are independent and additive risk factors of fatal outcome. Eur J Clin Microbiol Infect Dis 31: 1157-1162, 2012.

10. Wittenhagen P, Kronborg G, Weis N, Nielsen H, Obel N, Pedersen SS and Eugen-Olsen J: The plasma level of soluble urokinase receptor is elevated in patients with Streptococcus pneumoniae bacteraemia and predicts mortality. Clin Microbiol Infect 10: 409-415, 2004.

11. Bastarache JA, Ware LB and Bernard GR: The role of the coagulation cascade in the continuum of sepsis and acute lung injury and acute respiratory distress syndrome. Semin Respir Crit Care Med 27: 365-376, 2006

12. Koch A, Voigt S, Kruschinski C, Sanson E, Dückers H, Horn A, Yagmur E, Zimmermann H, Trautwein C and Tacke F: Circulating soluble urokinase plasminogen activator receptor is stably elevated during the first week of treatment in the intensive care unit and predicts mortality in critically ill patients. Crit Care 15: R63, 2011.

13. Casagranda I, Vendramin C, Callegari T, Vidali M, Calabresi A, Ferrandu G, Cervellin G, Cavazza M, Lippi G, Zanotti I, et al: Usefulness of suPAR in the risk stratification of patients with sepsis admitted to the emergency department. Intern Emerg Med 10: 725-730, 2015

14. Geboers DG, de Beer FM, Tuip-de Boer AM, van der Poll T, Horn J, Cremer OL, Bonten MJ, Ong DS, Schultz MJ and Bos LD: Plasma suPAR as a prognostic biological marker for ICU mortality in ARDS patients. Intensive Care Med 41: $1281-1290,2015$

15. Levy MM, Fink MP, Marshall JC, Abraham E, Angus D, Cook D, Cohen J, Opal SM, Vincent JL and Ramsay G; International Sepsis Definitions Conference: 2001 SCCM/ESICM/ACCP/ATS/SIS international sepsis definitions conference. Intensive Care Med 29: 530-538, 2003.

16. ARDS Definition Task Force; Ranieri VM, Rubenfeld GD, Thompson BT, Ferguson ND, Caldwell E, Fan E, Camporota L and Slutsky AS: Acute respiratory distress syndrome: The Berlin Definition. JAMA 307: 2526-2533, 2012.
17. Vincent JL, Moreno R, Takala J, Willatts S, De Mendonca A, Bruining H, Reinhart CK, Suter PM and Thijs LG: The SOFA (Sepsis-related Organ Failure Assessment) score to describe organ dysfunction/failure. On behalf of the Working Group on Sepsis-Related Problems of the European Society of Intensive Care Medicine. Intensive Care Med 22: 707-710, 1996.

18. Knaus WA, Zimmerman JE, Wagner DP, Draper EA and Lawrence DE: APACHE-acute physiology and chronic health evaluation: A physiologically based classification system. Crit Care Med 9: 591-597, 1981.

19. Eugen-Olsen J, Gustafson P, Sidenius N, Fischer TK, Parner J, Aaby P, Gomes VF and Lisse I: The serum level of soluble urokinase receptor is elevated in tuberculosis patients and predicts mortality during treatment: A community study from Guinea-Bissau. Int J Tuberc Lung Dis 6: 686-692, 2002.

20. Kofoed K, Eugen-Olsen J, Petersen J, Larsen K and Andersen O: Predicting mortality in patients with systemic inflammatory response syndrome: An evaluation of two prognostic models, two soluble receptors, and a macrophage migration inhibitory factor. Eur J Clin Microbiol Infect Dis 27: 375-383, 2008.

21. Ostrowski SR, Ullum H, Goka BQ, Høyer-Hansen G, Obeng-Adjei G, Pedersen BK, Akanmori BD and Kurtzhals JA: Plasma concentrations of soluble urokinase-type plasminogen activator receptor are increased in patients with malaria and are associated with a poor clinical or a fatal outcome. J Infect Dis 191: 1331-1341, 2005.

22. Pliyev BK: Activated human neutrophils rapidly release the chemotactically active D2D3 form of the urokinase-type plasminogen activator receptor (uPAR/CD87). Mol Cell Biochem 321: 111-122, 2009.

23. Sidenius N, Sier CF, Ullum H, Pedersen BK, Lepri AC, Blasi F and Eugen-Olsen J: Serum level of soluble urokinase-type plasminogen activator receptor is a strong and independent predictor of survival in human immunodeficiency virus infection. Blood 96: 4091-4095, 2000.

24. Ostergaard C, Benfield T, Lundgren JD and Eugen-Olsen J: Soluble urokinase receptor is elevated in cerebrospinal fluid from patients with purulent meningitis and is associated with fatal outcome. Scand J Infect Dis 36: 14-19, 2004.

25. Mondino A and Blasi F: uPA and uPAR in fibrinolysis, immunity and pathology. Trends Immunol 25: 450-455, 2004.

26. Rijneveld AW, Levi M, Florquin S, Speelman P, Carmeliet P and van Der Poll T: Urokinase receptor is necessary for adequate host defense against pneumococcal pneumonia. J Immunol 168 : 3507-3511, 2002

27. Park YJ,Liu G, Tsuruta Y,Lorne E and Abraham E: Participation of the urokinase receptor in neutrophil efferocytosis. Blood 114: 860-870, 2009

28. Wiersinga WJ, Kager LM, Hovius JW, van der Windt GJ, de Vos AF, Meijers JC, Roelofs JJ, Dondorp A, Levi M, Day NP, et al: Urokinase receptor is necessary for bacterial defense against pneumonia-derived septic melioidosis by facilitating phagocytosis. J Immunol 184: 3079-3086, 2010.

29. Slot O, Brunner N, Locht H, Oxholm P and Stephens RW: Soluble urokinase plasminogen activator receptor in plasma of patients with inflammatory rheumatic disorders: Increased concentrations in rheumatoid arthritis. Ann Rheum Dis 58: 488-492, 1999.

30. Pliyev BK and Menshikov MY: Release of the soluble urokinase-type plasminogen activator receptor (suPAR) by activated neutrophils in rheumatoid arthritis. Inflammation 33: $1-9,2010$.

31. Garcia-Monco JC, Coleman JL and Benach JL: Soluble urokinase receptor (UPAR, CD 87) is present in serum and cerebrospinal fluid in patients with neurologic diseases. J Neuroimmunol 129: 216-223, 2002.

32. Yilmaz G, Mentese A, Kaya S, Uzun A, Karahan SC and Koksal I: The diagnostic and prognostic significance of soluble urokinase plasminogen activator receptor in Crimean-Congo hemorrhagic fever. J Clin Virol 50: 209-211, 2011.

33. Backes Y, van der Sluijs KF, Tuip de Boer AM, Hofstra JJ, Vlaar AP, Determann RM, Knape P, Mackie DP and Schultz MJ: Soluble urokinase-type plasminogen activator receptor levels in patients with burn injuries and inhalation trauma requiring mechanical ventilation: An observational cohort study. Crit Care 15: R270, 2011

34. Wang H, Yang T, Li D, Wu Y, Zhang X, Pang C, Zhang J, Ying B, Wang T and Wen F: Elevated circulating PAI-1 levels are related to lung function decline, systemic inflammation, and small airway obstruction in chronic obstructive pulmonary disease. Int J Chron Obstruct Pulmon Dis 11: 2369-2376, 2016. 
35. Portelli MA, Moseley C, Stewart CE, Postma DS, Howarth P, Warner JA, Holloway JW, Koppelman GH, Brightling C and Sayers I: Airway and peripheral urokinase plasminogen activator receptor is elevated in asthma and identifies a severe, nonatopic subset of patients. Allergy 72: 473-482, 2017.

36. Hamie L, Daoud G, Nemer G, Nammour T, El Chediak A, Uthman IW, Kibbi AG, Eid A and Kurban M: SuPAR, an emerging biomarker in kidney and inflammatory diseases. Postgrad Med J 94: 517-524, 2018.

37. Pehlivanlar-Kucuk M, Kucuk AO, Ozturk CE, Er MC and Ulger F: The association between serum uric acid level and prognosis in critically Ill patients, uric acid as a prognosis predictor. Clin Lab 64: 1491-1500, 2018.
38. Donadello K, Scolletta S, Covajes C and Vincent JL: suPAR as a prognostic biomarker in sepsis. BMC Med 10: 2, 2012.

(c) (i) () $९$ This work is licensed under a Creative Commons

EY No No Attribution-NonCommercial-NoDerivatives 4.0 International (CC BY-NC-ND 4.0) License. 